\title{
Research of digital detection method based on model
}

\author{
Jing $\mathrm{Li}^{1, \mathrm{a}}$, Dan-Ya Zhang ${ }^{2, \mathrm{~b}, \dagger}$ and Zhen-Yu Li ${ }^{3, \mathrm{c}}$

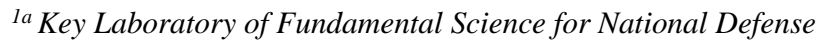 \\ of Aeronautical Digital Manufacturing Process, Shenyang Aerospace University, \\ Shenyang, China. 110136ddress of first author, including country \\ alijingmessi@163.com, bzhangdanyal@126.com, ${ }^{c} l v 0527 @ 163 . c o m$ \\ ${ }^{\dagger}$ Jing $\mathrm{Li}$
}

\begin{abstract}
With the development of digital design and manufacturing technology of aircraf, The large-scale overall structural element amount used unceasing increase, as well as the civil aircraft efficiency and the secure request daily enhancement, also proposed to the product manufacture precision a higher request, causes the product examination difficulty to be day by day big, thus to surveyed the technology to propose the new demand, The traditional survey technology has satisfied the airplane spare part with difficulty fast, highly effective, the high accuracy examination request. Current has become based on the three-dimensional model digitized examination technology application makes a connection the airplane complex components and the great size spare part digitization design, the manufacture, the examination integration flow, the promotion examination efficiency and the level key link.
\end{abstract}

Keywords: Model; Digital; Detection.

\section{Introduction}

\section{Technical principle}

The digitized examination technology basic principle is uses on the examination software read components the geometry characteristic which treats the examination information and is connected (surface, line, spot, circle and so on), obtains treats measures the essential factor the digital quantity theoretical value (including a composition geometry essential factor all vector coordinate figure as well as vector value), Then put the parts to be tested in the measurement space, it allows use of the geometric elements of the digital quantity of the theoretical value drive three coordinate measuring machine to measure actual parts, parts to be tested is obtained on the surface of each point in space coordinates, the coordinates data, simplify, denoising by fitting and so on a series of processing and analysis, The fitting forms the corresponding geometry element, like the straight line, the plane, the circle, the circular cone, the circular slot and so on, obtain its shape, the location tolerance and other geometry 
quantity data again after the mathematical computation method, then carries on the contrast with the primitive three dimensional numerical model in digital quantity theoretical value, thus analyzes on the components each geometry essential factor ultra difference situation, completes the correlation the examination duty.

\section{Equipment principle}

At present in the aviation enterprise, the computer secondary measuring system main check-out facility has the coordinate measuring machines, the laser scanner, the laser tracker and the phantom scanner, the this article main research based on the coordinate measuring machines digitized examination technology. the basic principle of measuring machine is measured parts into it allows measuring space, accurately measure points on the surface of the parts to be tested in the space of three coordinates numerical, will these values through computer data processing, the coordinates of point fitting form measuring elements, such as round, ball, cylindrical, cone and curved surface and so on, as shown in figure1, through the mathematical calculation method of its shape and position tolerance and other geometrical quantity data.

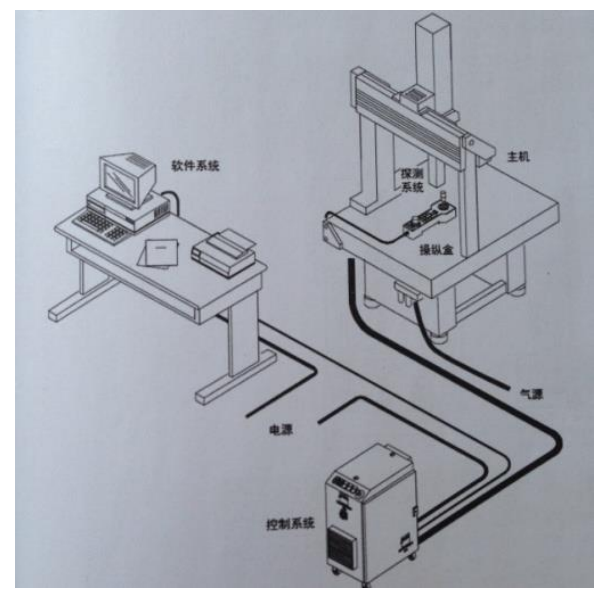

Figure 1 measuring machine structure diagram

\section{Examination type}

\section{Import sample model into the PC software DMIS/Planner.}

Set the test parameters, the machine information, side header, compensation. Coordinates measuring engine when survey components, is uses the marking pin the gem ball with to measure the parts list surface contact, the contact point 
coordinates and the system transmission gem ball central point coordinates differ a gem ball radius, needs marking pin radius value which obtains through the verification, to measurement result revision, In the process of measurement, often need to pass a different side of the head Angle, length and diameter of combination to complete the measurement task. Different position of measurement point must be after conversion to the same coordinate calculation, so need to pass to side head to check for the location of the relationship between different side head Angle. Side head calibration principle for through on a recognized standard of measuring points to get the lateral head of the diameter and position of real relationship. Generally USES the standard is a standard ball (ball degree is less than 0.1 microns), in the calibration check on the standard ball side of the head, measuring software first transfer according to the measuring system of coordinates of measuring points fitting calculation of a ball, calculate the diameter of the fitting of the ball and standard cue ball point coordinates. The fitting of the ball diameter minus the diameter of the standard ball, is the correction of the equivalent diameter of lateral head (needle).

\section{The establishment of coordinate system}

The components must have to have the correct attire before the examination to clamp, the enough examination space and the constant temperature space, similarly also must have to have a measuring engine programmer to carry on the operation. The coordinate system establishment is the following survey foundation, will establish the wrong coordinate system directly to cause the survey size the mistake, therefore established a correct reference direction will be the coordinate system is extremely essential and important.

This paper USES is 321 the establishment of coordinate method, fine coordinate system. To establish the principle of coordinate system is divided into three steps:

(1) Part is be right find . (2)The axis of rotation, (3) CAD = artifacts

\section{Detection.}

In this paper, we measure the two characteristics of samples and the cylindricity of cylinder 1 and 1 plane on flatness. 


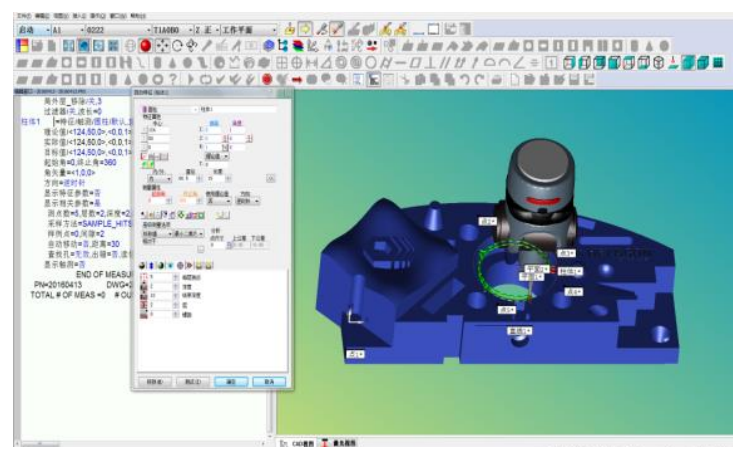

Figure 2 barrel 1 automatic survey

\section{Evaluation result output}

Automatically execute a program, measuring machine, hand-held measuring machine control handle, observing on the probe points, to prevent accidental firing pin, after the program execution, size evaluation. Open the size report view, as shown.

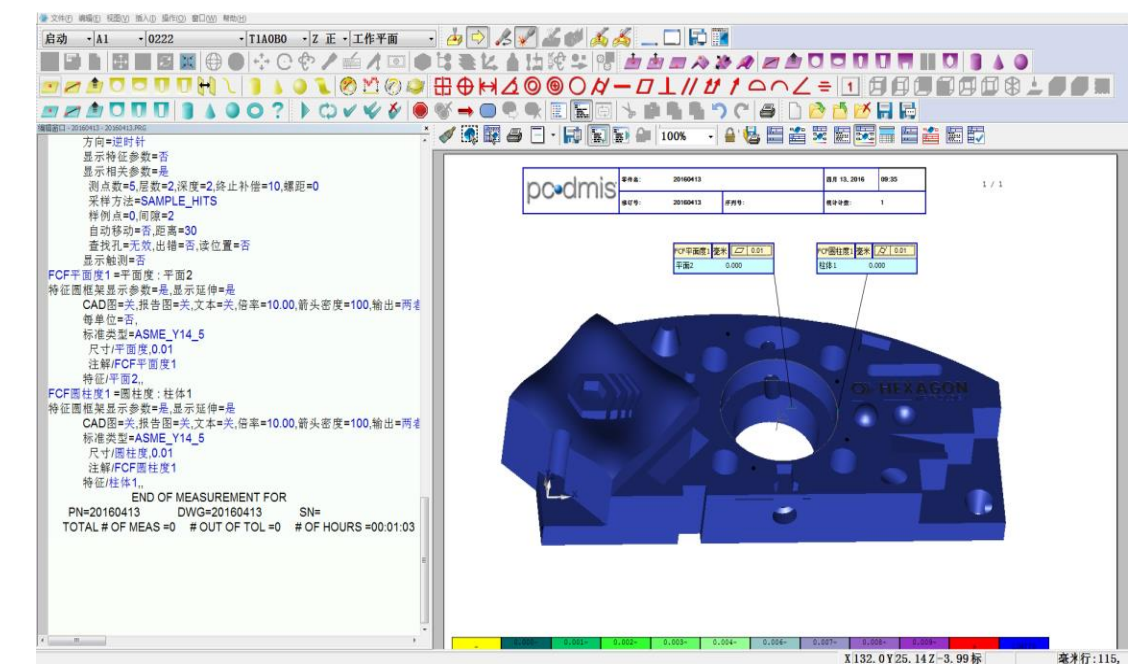

Figure 3 size report window

In the report to the right view window display mode (cad model), we can see the work piece title bar, including LOGO, parts and working time and figure label can change according to the need of customers, open report window, Obvious may see the upper deviation, the lower deviation, as well as tolerance zone, if behind transect demonstration for red, then is the ultra difference, green namely for qualified. Because this article chooses is a standard type examination, 
therefore the examination result is qualified. If is unqualified, discovers the unqualified spot, carries on examines repeatedly, analyzes the ultra difference reason, the ultra difference reason has very many kinds, for instance the coordinate system establishment, picks too few, work piece place and so on.

Figure 4 Size evaluation

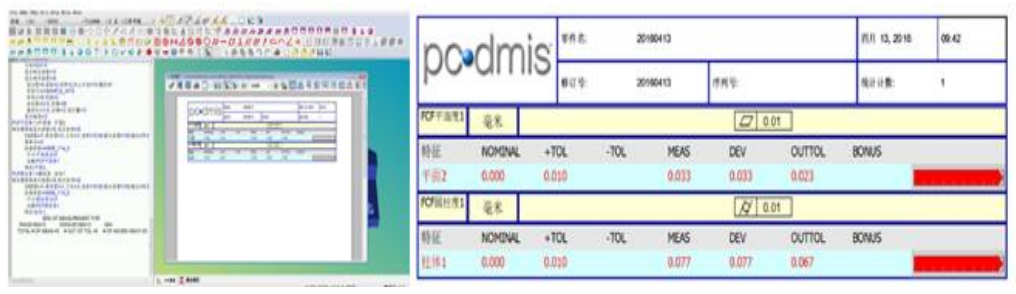

\section{Summary}

At present, the digital detection based on MBD technology is still in the early stage of development, the lack of a complete system, feasible digital detection technology and implementing scheme, digital detection in the process there is no uniform standard, Cause MBD digital detection technique and its application in manufacturing enterprises in China is very limited. Based on the outcome of the three-coordinate measurement technology research at home and abroad on the basis of combining the detection status and demands of an Aviation Enterprise.

\section{Acknowledgement}

This research was financially supported by the National Science Foundation.

\section{References}

[1] KUMARA, FUNJ, KOWT. An automated design and assembly of interference free modular fixture setup [J]. Computer Aided Design, 2000, 32(10): 583-586.

[2] WUY, RONGY, MAW, et al. Automated modular fixture planning: accuracy, clamping, and accessibility analysis [J]. Robotics \& Computer-Integrated Manufacturing, 1998, 14(1): 16-26.

[3] Michalewicz Z, et al. Genetic Algorithms and Optimal Control Problem. In: Proc. Of 29th IEEE Conf. on Decision and Control [J]. 1990. 1664-1666.

[4] H A EIMARAGHY, P H Gu. Expert system for inspection planning, Annals of the CIRP 1987, 37(1): 85-89.

[5] WEVERSHEIM, J AUGE. Automatic generation of part programs for CNC-coordinate measuring machines linked to CAD/CAM systems. Annals of the CIRP, 1986, 35(1): 341-345. 